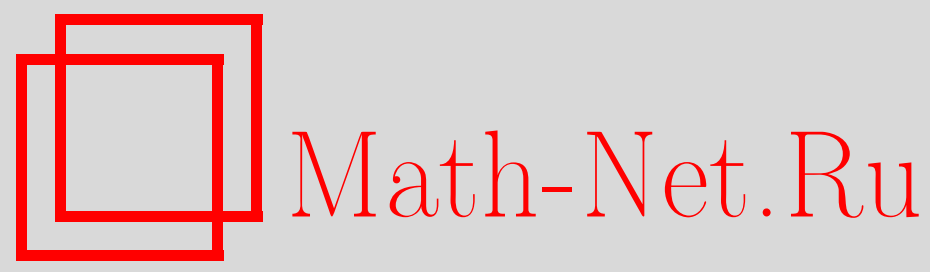

Е. Е. Бердышева, Оптимальное множество модуля непрерывности в точном неравенстве Джексона в пространстве $L_{2}$, Матем. заметки, 2004, том 76, выпуск 5, 666-674

DOI: https://doi.org/10.4213/mzm137

Использование Общероссийского математического портала Math-Net.Ru подразумевает, что вы прочитали и согласны с пользовательским соглашением http://www.mathnet.ru/rus/agreement

Параметры загрузки:

IP : 54.164 .48 .24

26 апреля 2023 г., $18: 23: 25$

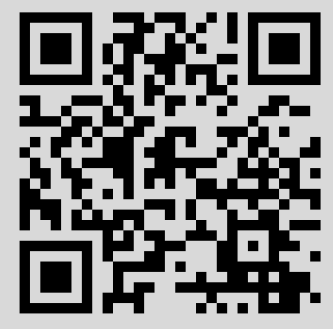


УДК 517.518.834

\section{ОПТИМАЛЬНОЕ МНОЖЕСТВО МОДУЛЯ НЕПРЕРЫВНОСТИ В ТОЧНОМ НЕРАВЕНСТВЕ ДЖЕКСОНА В ПРОСТРАНСТВЕ $L_{2}$ Е. Е. Бердышева}

Функции $f \in L_{2}[-\pi, \pi]$ и компактному множеству $Q \subset[-\pi, \pi]$ сопоставим величину $\omega(f, Q)=\sup _{t \in Q}\|f(\cdot+t)-f(\cdot)\|_{L_{2}[-\pi, \pi]}$, являющуюся аналогом модуля непрерьвности. Обозначим через $K(n, Q)$ наименьшую константу в неравенстве Джексона между наилучшим приближением функции $f$ тригонометрическими полиномами степени $n-1$ в пространстве $L_{2}[-\pi, \pi]$ и модулем непрерывности $\omega(f, Q)$. Из результатов Н. И. Черных следует, что $K(n, Q) \geqslant 1 / \sqrt{2}$ и $K(n,[0, \pi / n])=1 / \sqrt{2}$. На основании одного результата В.А. Юдина мы показьваем, что если мера множества $Q$ меньше, чем $\pi / n$, то $K(n, Q)>1 / \sqrt{2}$.

Библиографоия: 13 названий.

Пусть $L_{2}=L_{2}[-\pi, \pi]$ есть пространство комплекснозначных $2 \pi$-периодических функций, суммируемых с квадратом на $[-\pi, \pi]$, рассматриваемое с обычной нормой

$$
\|f\|_{L_{2}}=\left(\frac{1}{2 \pi} \int_{-\pi}^{\pi}|f(x)|^{2} d x\right)^{1 / 2}
$$

Пусть $T_{n}$ есть множество тригонометрических полиномов

$$
g(t)=\sum_{k=-n}^{n} c_{k} e^{i k t}
$$

степени не более $n$ с комплексными коэффициентами. Для функции $f \in L_{2}$ обозначим через

$$
E_{n}(f)=\inf \left\{\|f-g\|_{L_{2}}: g \in T_{n-1}\right\}
$$

наилучшее приближение функции $f$ тригонометрическими полиномами порядка $n-1$. Для $\delta>0$ величина

$$
\omega(f,[0, \delta])=\sup \left\{\|f(\cdot+t)-f(\cdot)\|_{L_{2}}: t \in[0, \delta]\right\}
$$

является классическим модулем непрерьвности первого порядка. Неравенством Джексона назьвается неравенство вида

$$
E_{n}(f) \leqslant K \omega(f,[0, \delta])
$$

Работа выполнена при финансовой поддержке фонда Александра фон Гумбольдта, стипендия RUS/1072237 STP, и Российского фонда фундаментальных исследований, грант № 02-01-00783. 
выполняющееся для всех функций $f \in L_{2}$ с константой $K$, не зависящей от $f$. Наименьшая константа в неравенстве Джексона

$$
K(n,[0, \delta])=\sup \left\{\frac{E_{n}(f)}{\omega(f,[0, \delta])}: f \in L_{2}\right\}
$$

назьвается константой Джексона; здесь и далее в аналогичных ситуациях мы полагаем, что $0 / 0=0$ и $c / 0=+\infty$ при $c>0$. Неравенство Джексона в различньх функциональных пространствах с различными модулями непрерьвности имеет богатейшую историю (см., например, [1], [2]). Одним из базовых в этой тематике является следующий результат Черных [3]-[5]:

$$
K(n,[0, \delta])=\frac{1}{\sqrt{2}}, \quad \frac{\pi}{n} \leqslant \delta \leqslant \pi, \quad K(n,[0, \delta])>\frac{1}{\sqrt{2}}, \quad 0<\delta<\frac{\pi}{n} .
$$

Оценка снизу

$$
K(n,[0, \delta]) \geqslant \frac{1}{\sqrt{2}}
$$

для всех $0<\delta \leqslant 2 \pi$ вытекает также из более общего результата В. И. Бердьшева [6].

При исследовании неравенства Джексона (2) Черных фактически решил следующую экстремальную задачу. Пусть $n$ - натуральное число, $\Phi(n)$ - класс функций вида

$$
\varphi(t)=\frac{a_{0}}{2}+\sum_{k=1}^{\infty} a_{k} \cos k t, \quad t \in[0, \pi]
$$

удовлетворяющих следующим двум свойствам:

1) $\varphi(t) \not \equiv 0 ; \varphi(t) \geqslant 0, t \in[0, \pi]$;

2) $a_{k}=\frac{2}{\pi} \int_{0}^{\pi} \varphi(t) \cos k t d t \leqslant 0, k \geqslant n$.

Для $\varphi \in \Phi(n)$ положим $\tau(\varphi)=\pi$, если $\varphi(\pi) \neq 0$, и

$$
\tau(\varphi)=\inf \{\tau>0: \varphi(x)=0 \text { при } \tau \leqslant x \leqslant \pi\}
$$

в противном случае. Обозначим

$$
m(n)=\inf \{\tau(\varphi): \varphi \in \Phi(n)\}, \quad n \in \mathbb{N} .
$$

Из результатов Черных [3]-[5] следует, что $m(n)=\pi / n, n \in \mathbb{N}$, и экстремальной является функция

$$
\Psi_{n}(t)= \begin{cases}\sin n t, & 0 \leqslant t \leqslant \frac{\pi}{n}, \\ 0, & \frac{\pi}{n} \leqslant t \leqslant \pi .\end{cases}
$$

Более тонкую в сравнении с (5) задачу рассмотрел Юдин [7]:

$$
M(n)=\inf \{\vartheta(\varphi): \varphi \in \Phi(n)\}, \quad \vartheta(\varphi)=\operatorname{meas}\{t \in[0, \pi]: \varphi(t)>0\}
$$


здесь и всюду в работе meas обозначает обычную лебеговскую меру на прямой. Юдин доказал [7], что

$$
M(n)=\frac{\pi}{n}, \quad n \in \mathbb{N},
$$

и функция (6) является экстремальной. Он также построил пример другой функции, экстремальной в задачах $m(n)$ и $M(n)$.

В этой работе мы изучаем следующее обобщение задачи (2), (3). Пусть $Q$ - компактное множество на $[-\pi, \pi]$. Введем модуль непрерывности

$$
\omega(f, Q)=\sup \left\{\|f(\cdot+t)-f(\cdot)\|_{L_{2}}: t \in Q\right\}
$$

Аналогично (2) и (3), будем рассматривать неравенство Джексона

$$
E_{n}(f) \leqslant K \omega(f, Q)
$$

и константу Джексона

$$
K(n, Q)=\sup \left\{\frac{E_{n}(f)}{\omega(f, Q)}: f \in L_{2}\right\}
$$

В силу свойства

$$
\|f(\cdot+t)-f(\cdot)\|_{L_{2}}=\|f(\cdot-t)-f(\cdot)\|_{L_{2}}
$$

имеет место равенство $\omega(f, Q)=\omega(f,|Q|)$, где $|Q|=\{|q|: q \in Q\} \subset[0, \pi]$. Таким образом, мы можем ограничиться рассмотрением компактов $Q \subset[0, \pi]$. Нетрудно видеть, что для компактов $Q_{1}$ и $Q_{2}$ на $[0, \pi]$ справедливо утверждение

$$
Q_{1} \subset Q_{2} \Longrightarrow K\left(n, Q_{1}\right) \geqslant K\left(n, Q_{2}\right)
$$

Из этого свойства и результата Черных (4) вытекает, что для любого $n \in \mathbb{N}$

$$
\min \{K(n, Q): Q-\text { компакт на }[0, \pi]\}=\frac{1}{\sqrt{2}}
$$

На основании результата Юдина (8), А. Г. Бабенко в работе [2] высказал предположение, что $K(n, Q)>1 / \sqrt{2}$ для любого замкнутого множества $Q \subset[0, \pi]$ c meas $Q<\pi / n$. В представленной работе мы доказьваем, что это предположение верно. Введем величину

$$
h(n)=\inf \left\{\operatorname{meas} Q: Q-\text { компакт на }[0, \pi], K(n, Q)=\frac{1}{\sqrt{2}}\right\} .
$$

Справедливо следующее утверж дение. 
Теорема 1. Имеет место равенство

$$
h(n)=\frac{\pi}{n} .
$$

Нижняя грань в (13) достигается на множестве $Q=[0, \pi / n]$.

При изучении величины (13) достаточно ограничиться компактами $Q$ положительной меры. Действительно, предположим, что нижняя грань в (13) достигается на множестве $Q$ меры нуль. Построим последовательность множеств $Q_{k}$ с мерой $1 / k$, содержащих $Q$. В силу (11) справедливо неравенство

$$
K\left(n, Q_{k}\right) \leqslant K(n, Q)=\frac{1}{\sqrt{2}} .
$$

С другой стороны, из (12) следует, что

$$
K\left(n, Q_{k}\right) \geqslant \frac{1}{\sqrt{2}} .
$$

Таким образом,

$$
K\left(n, Q_{k}\right)=\frac{1}{\sqrt{2}},
$$

и мы получаем, что нижняя грань в (13) есть нуль, даже если в определении величины (13) мы рассматриваем только множества положительной меры.

Как хорошо известно, в терминах коэффицциентов Фурье

$$
\widehat{f_{k}}=\frac{1}{2 \pi} \int_{-\pi}^{\pi} f(t) e^{-i k t} d t
$$

функции $f$ выражения для величин (1) и (9) выглядят следующим образом:

$$
E_{n}^{2}(f)=\sum_{|k| \geqslant n}\left|\widehat{f}_{k}\right|^{2}, \quad \omega^{2}(f, Q)=2 \sup _{q \in Q} \sum_{k \in \mathbb{Z}}\left|\widehat{f}_{k}\right|^{2}(1-\cos k q) .
$$

Положим $a_{0}=\left|\widehat{f}_{0}\right|^{2}, a_{k}=\left|\widehat{f}_{k}\right|^{2}+\left|\widehat{f}_{-k}\right|^{2}, k \in \mathbb{N}$. Тогда

$$
E_{n}^{2}(f)=\sum_{k=n}^{\infty} a_{k}, \quad \omega^{2}(f, Q)=2 \sup _{q \in Q} \sum_{k=1}^{\infty} a_{k}(1-\cos k q) \geqslant 2 \sup _{q \in Q} \sum_{k=n}^{\infty} a_{k}(1-\cos k q) .
$$

Отсюда следует, что

$$
2 K^{2}(n, Q)=\sup \left\{\frac{\sum_{k \geqslant n} a_{k}}{\sup _{q \in Q} \sum_{k \geqslant n} a_{k}(1-\cos k q)}: a_{k} \geqslant 0, \sum_{k \geqslant n} a_{k}<\infty\right\} .
$$

Пусть $\mathscr{M}^{+}(Q)$ есть множество вещественных неотрицательных борелевских мер $\mu$ на компакте $Q$, нормированных условием

$$
\mu(Q)=\int_{Q} d \mu(t)=1 .
$$


Обозначим

$$
c_{k}(\mu)=\frac{2}{\pi} \int_{Q} \cos k t d \mu(t), \quad \mu \in \mathscr{M}^{+}(Q), \quad k \in \mathbb{Z}_{+} .
$$

В наших рассуждениях мы будем использовать две экстремальные величины:

$$
\begin{gathered}
\eta(n, \mu)=\sup _{k \geqslant n} c_{k}(\mu), \\
J(n, Q)=\frac{\pi}{2} \cdot \inf _{\mu \in \mathcal{M}^{+}(Q)} \eta(n, \mu) .
\end{gathered}
$$

Юдин [7] доказал следующую лемму (здесь мы записьваем ее в наших терминах).

Лемма 1 (В.А. Юдин [7]). Пусть $\sigma$ - неотрицательная мера Бореля на отрез$\kappa е[0, \pi]$. Предположим, что существует конечное множество $B \subset \mathbb{Z}_{+}$такое, что $c_{k}(\sigma) \leqslant 0$ при $k \in \mathbb{Z}_{+} \backslash$ В. Тогда числовой ряд

$$
\frac{c_{0}(\sigma)}{2}+\sum_{k=1}^{\infty} c_{k}(\sigma)
$$

абсолютно сходится.

Изучим свойства величины (15).

ЛЕмма 2. Пусть $Q$ - компактное множество положительной меры на отрезке $[0, \pi]$. Имеют место неравенства

$$
0 \leqslant J(n, Q)<1
$$

ДокАЗАТЕЛЬСТво. Из нормировки (14) следует, что для любой меры $\mu \in \mathscr{M}^{+}(Q)$ и любого $k \in \mathbb{Z}_{+}$имеет место неравенство $c_{k}(\mu) \leqslant 2 / \pi$. Отсюда вытекает, что $J(n, Q) \leqslant 1$. Докажем, что, на самом деле, имеет место строгое неравенство. Достаточно привести пример меры $\mu \in \mathscr{M}^{+}(Q)$, для которой $\eta(n, \mu)<2 / \pi$. Рассмотрим меру $\mu_{Q} \in \mathscr{M}^{+}(Q)$, заданную следующим образом: для борелевского множества $A \subseteq Q$ положим $\mu_{Q}(A)=$ meas $A /$ meas $Q$. Докажем, что для нее вьполняются неравенства $\eta\left(n, \mu_{Q}\right)<2 / \pi, n \in \mathbb{N}$. Мы должны оценить

$$
c_{k}\left(\mu_{Q}\right)=\frac{2}{\pi} \frac{1}{\operatorname{meas} Q} \int_{Q} \cos k t d t .
$$

Покажем, что $c_{k}\left(\mu_{Q}\right)<2 / \pi$ для всех $k \geqslant 1$. Зафиксируем $k$ и выберем положительное число $\varepsilon<\operatorname{meas} Q$. Пусть $t_{\nu}=2 \pi \nu / k, \nu=0,1, \ldots,[k / 2],-$ множество точек $t$ из [0, $\left.\pi\right]$, в которых функция cos $k t$ принимает свое наибольшее значение, равное единище. Положим

$$
Q_{\varepsilon}=Q \cap\left\{\bigcup_{\nu=0}^{l}\left[t_{\nu}-\frac{\varepsilon}{2(l+1)}, t_{\nu}+\frac{\varepsilon}{2(l+1)}\right]\right\}, \quad l=\left[\frac{k}{2}\right] .
$$

Имеем meas $Q_{\varepsilon} \leqslant \varepsilon$ и meas $\left(Q \backslash Q_{\varepsilon}\right)>0$. На множестве $Q \backslash Q_{\varepsilon}$ вьполняется неравенство $\cos k t \leqslant \beta$ с некоторьп $\beta<1$. Следовательно,

$$
\begin{aligned}
c_{k}\left(\mu_{Q}\right) & =\frac{2}{\pi} \frac{1}{\operatorname{meas} Q}\left(\int_{Q_{\varepsilon}} \cos k t d t+\int_{Q \backslash Q_{\varepsilon}} \cos k t d t\right) \\
& \leqslant \frac{2}{\pi} \frac{1}{\operatorname{meas} Q}\left(\operatorname{meas} Q_{\varepsilon}+\beta \cdot \operatorname{meas}\left(Q \backslash Q_{\varepsilon}\right)\right)<\frac{2}{\pi} .
\end{aligned}
$$


Итак, $c_{k}\left(\mu_{Q}\right)<2 / \pi$ для всех $k \geqslant 1$. Кроме того,

$$
\lim _{k \rightarrow \infty} c_{k}\left(\mu_{Q}\right)=0
$$

как предел коэффициентов Фурье функции $(\operatorname{meas} Q)^{-1} \cdot \chi_{Q}$, определенной и суммируемой на $[0, \pi]\left(\chi_{Q}\right.$ обозначает характеристическую функцию множества $\left.Q\right)$. Отсюда следует, что для любого $n \in \mathbb{N}$ выполняется неравенство

$$
\sup _{k \geqslant n} c_{k}\left(\mu_{Q}\right)<\frac{2}{\pi}
$$

Теперь докажем первое неравенство в (16). Предположим противное, т.е. предположим, что существует мера $\mu \in \mathscr{M}^{+}(Q)$, для которой $\eta(n, \mu)=\alpha<0$. Это означает, что $\mu$ удовлетворяет условиям леммы 1 с $B=\{0,1, \ldots, n-1\}$. В силу леммы 1 ряд $c_{0}(\mu) / 2+\sum_{k=1}^{\infty} c_{k}(\mu)$ должен сходиться. Но это невозможно, так как мы имеем оценку $c_{k}(\mu) \leqslant \alpha<0$ при $k \geqslant n$. Полученное противоречие доказывает неравенство $J(n, Q) \geqslant 0$.

В следующей лемме указана связь задач (15) и (10).

Лемма 3. Пусть $n \in \mathbb{N}, Q$ - компактное множество положительной меры на отрезке $[0, \pi]$. Имеет место соотношение

$$
2 K^{2}(n, Q)=\frac{1}{1-J(n, Q)} .
$$

При этом существует мера $\bar{\mu} \in \mathscr{M}^{+}(Q)$, на которой достигается нижняя грань в задаче (15).

Соотношение двойственности типа (17) впервые было замечено Арестовым (см. [8]). В различных ситуациях оно было доказано Бабенко, Арестовьм и автором [8]-[11]. Все эти результаты получены по одной и той же схеме. Ниже для полноты изложения мы приводим доказательство в нашем случае.

ДокаЗАТЕЛЬСТво. Рассмотрим множество функций

$$
\Omega(n)=\left\{\Delta(t)=\sum_{k \geqslant n} a_{k}(1-\cos k t): a_{k} \geqslant 0, \sum_{k \geqslant n} a_{k}=1\right\} .
$$

Легко видеть, что

$$
\begin{aligned}
2 K^{2}(n, Q) & =\sup \left\{\frac{1}{\sup _{t \in Q} \sum_{k \geqslant n} a_{k}(1-\cos k t)}: a_{k} \geqslant 0, \sum_{k \geqslant n} a_{k}=1\right\} \\
& =\sup _{\Delta \in \Omega(n)} \frac{1}{\|\Delta\|_{C(Q)}}=\frac{1}{R(n, Q)},
\end{aligned}
$$

где

$$
R(n, Q)=\inf _{\Delta \in \Omega(n)}\|\Delta\|_{C(Q)}
$$


есть расстояние от функции, равной тождественному нулю, до вьпуклого множества $\Omega(n)$ в пространстве $C(Q)$. В силу известной теоремы двойственности (см., например, $[1$, п. 1.3]) и теоремы Рисса об общем виде линейного непрерьвного функционала в пространстве $C$ на компакте (см., например, [12, п. IV .6.3])

$$
\begin{aligned}
R(n, Q) & =\sup \left\{\inf _{\Delta \in \Omega(n)} w(\Delta): w \in C^{*}(Q),\|w\|_{C^{*}(Q)} \leqslant 1\right\} \\
& =\sup _{\mu \in \mathcal{M}(Q)} \inf _{\Delta \in \Omega(n)} \int_{Q} \Delta(t) d \mu(t),
\end{aligned}
$$

где $\mathscr{M}(Q)$ есть множество борелевских вещественных вероятностных мер $\mu$ на $Q$. При этом существует мера $\bar{\mu} \in \mathscr{M}(Q)$, на которой достигается супремум в (18). Поскольку все функции $\Delta \in \Omega(n)$ неотрицательны, мы можем ограничиться в (18) рассмотрением только мер $\mu \in \mathscr{M}^{+}(Q)$. Таким образом,

$$
R(n, Q)=\sup _{\mu \in \mathscr{M}^{+}(Q)} \inf _{\Delta \in \Omega(n)} \int_{Q} \Delta(t) d \mu(t)
$$

Для каждой меры $\mu \in \mathscr{M}^{+}(Q)$ имеем

$$
\begin{aligned}
\inf _{\Delta \in \Omega(n)} \int_{Q} \Delta(t) d \mu(t) & =\inf \left\{\int_{Q} \sum_{k \geqslant n} a_{k}(1-\cos k t) d \mu(t): a_{k} \geqslant 0, \sum_{k \geqslant n} a_{k}=1\right\} \\
& =\inf \left\{\sum_{k \geqslant n} a_{k} \int_{Q}(1-\cos k t) d \mu(t): a_{k} \geqslant 0, \sum_{k \geqslant n} a_{k}=1\right\} \\
& =\inf _{k \geqslant n} \int_{Q}(1-\cos k t) d \mu(t)=1-\sup _{k \geqslant n} \int_{Q} \cos k t d \mu(t) \\
& =1-\sup _{k \geqslant n} \frac{\pi}{2} \cdot c_{k}(\mu)=1-\eta(n, \mu) .
\end{aligned}
$$

Следовательно,

$$
R(n, Q)=\sup _{\mu \in \mathscr{M}^{+}(Q)}(1-\eta(n, \mu))=1-J(n, Q) .
$$

Лемма доказана.

ЗАмечание 1 . Если meas $Q>0$, то константа $K(n, Q)$ конечна. Это вытекает из соотношения двойственности (17) и правого неравенства в (16).

ЗАмЕчАнИЕ 2. Соотношение двойственности (17) и левое неравенство в (16) дают еще одно доказательство формулы (12).

В следующей лемме мы укажем связь задач (13) и (7).

Лемма 4. Имеет место равенство $h(n)=M(n)$. 
ДокАЗАТЕльСтво. Сначала докажем неравенство

$$
h(n) \geqslant M(n) .
$$

Пусть $Q$ - компакт на $[0, \pi]$ такой, что $K(n, Q)=1 / \sqrt{2}$ (например, $Q=[0, \pi / n])$. Из (17) следует, что $J(n, Q)=0$. Пусть $\bar{\mu} \in \mathscr{M}^{+}(Q)$ есть экстремальная мера задачи (15). Определим функцию $\bar{\varphi}(t)$ как сумму абсолютно сходящегося (в силу леммы 1$)$ ряда

$$
\bar{\varphi}(t)=\frac{c_{0}(\bar{\mu})}{2}+\sum_{k=1}^{\infty} c_{k}(\bar{\mu}) \cos k t, \quad t \in[0, \pi] .
$$

Функция $\bar{\varphi}$ есть непрерывная четная $2 \pi$-периодическая функция. Она задает на $[0, \pi]$ борелевскую меру $\bar{\psi}$ по формуле

$$
\bar{\psi}(A)=\int_{A} \bar{\varphi}(t) d t
$$

для (борелевского) множества $A \subseteq[0, \pi]$. Расширим меру $\bar{\mu}$, заданную на $Q$, до борелевской меры $\overline{\bar{\mu}}$ на $[0, \pi]$, положив $\overline{\bar{\mu}}(A)=\bar{\mu}(A \cap Q)$ на борелевских множествах $A \subseteq[0, \pi]$. Эта мера обращается в нуль вне множества $Q$, совпадает с $\bar{\mu}$ на $Q$ и неотрицательна на $[0, \pi]$. Для нее мы имеем

$$
c_{k}(\overline{\bar{\mu}})=\frac{2}{\pi} \int_{0}^{\pi} \cos k t d \overline{\bar{\mu}}(t)=\frac{2}{\pi} \int_{Q} \cos k t d \bar{\mu}(t)=c_{k}(\bar{\mu})=c_{k}(\bar{\psi}) .
$$

По теореме единственности для коэффициентов Фурье-Стилтьеса (см. [13, гл. VII, §1]) имеем $\overline{\bar{\mu}}=\bar{\psi}$. Следовательно, функция $\bar{\varphi}$ неотрицательна на $[0, \pi]$ и обрашается в нуль вне множества $Q$. Кроме того, $c_{k}(\bar{\mu}) \leqslant 0, k \geqslant n$, в силу равенства $\eta(n, \bar{\mu})=0$. Таким образом, $\bar{\varphi} \in \Phi(n)$. Для множества $\bar{Q}=\operatorname{supp} \bar{\varphi} \subseteq Q$ также будем иметь $K(n, \bar{Q})=1 / \sqrt{2}$. Далее, meas $Q \geqslant \operatorname{meas} \bar{Q} \geqslant M(n)$ в силу определения (7). Минимизируя неравенство meas $Q \geqslant M(n)$ по всем компактам $Q$ со свойством $K(n, Q)=1 / \sqrt{2}$, получаем неравенство (19).

Докажем теперь противоположное неравенство

$$
h(n) \leqslant M(n) .
$$

Возьмем $\varepsilon>0$. Существует функция $\widetilde{\varphi} \in \Phi(n)$, для которой

$$
\operatorname{meas}\{t \in[0, \pi]: \widetilde{\varphi}(t)>0\}<M(n)+\varepsilon .
$$

Положим $\widetilde{Q}=\operatorname{supp} \widetilde{\varphi}$. Имеем meas $\widetilde{Q}<M(n)+\varepsilon$. Определим борелевскую меру $\widetilde{\mu}$ на $\widetilde{Q}$ по следующему правилу: для борелевского множества $A \subseteq \widetilde{Q}$ положим

$$
\widetilde{\mu}(A)=\left(\int_{0}^{\pi} \widetilde{\varphi}(\tau) d \tau\right)^{-1} \cdot \int_{A} \widetilde{\varphi}(\tau) d \tau .
$$


Так как $\widetilde{\varphi} \geqslant 0$, то $\widetilde{\mu} \in \mathscr{M}^{+}(Q)$. При этом

$$
c_{k}(\widetilde{\mu})=\left(\int_{0}^{\pi} \widetilde{\varphi}(\tau) d \tau\right)^{-1} \cdot \frac{2}{\pi} \int_{A} \widetilde{\varphi}(t) \cos k t d t .
$$

По определению класса $\Phi(n)$ имеем $\eta(n, \widetilde{\mu}) \leqslant 0$. Учитьвая левое неравенство в $(16)$, получаем $J(n, \widetilde{Q})=0$. Следовательно, $K(n, \widetilde{Q})=1 / \sqrt{2}$ и $h(n) \leqslant \operatorname{meas} \widetilde{Q}<M(n)+\varepsilon$. Так как $\varepsilon>0$ может быть выбрано произвольньг, отсюда следует неравенство (20). Лемма доказана.

Утверждение теоремы вытекает теперь из леммы 4 и результата Юдина (8).

Выражаю глубокую признательность В.В. Арестову за постоянньй контакт по теме работы. Благодарю А.Г. Бабенко, который обратил мое внимание на результат В.А. Юдина (8) и высказал предположение о связи этого результата со свойствами константы Джексона. Благодарю обоих за замечания, относяшиеся к оформлению работы.

\section{СПИСОК ЦИТИРОВАННОЙ ЛИТЕРАТУРЫ}

[1] Корнейчук Н. П. Точные константы в теории приближения. М.: Наука, 1987.

[2] Babenko A. G. On the Jackson-Stechkin inequality for the best $L^{2}$-approximations of functions by trigonometric polynomials // Proc. Steklov. Inst. Mat. Suppl. 1. 2001. P. S30-S47.

[3] Черных Н. И. О неравенстве Джексона в $L_{2} / /$ Тр. МИАН. 1967. Т. 88. С. 71-74.

[4] Черных Н.И. О наилучшем приближении периодических функций тригонометрическими полиномами в $L_{2} / /$ Матем. заметки. 1967. Т. 2. № 5. С. 513-522.

[5] Arestov V. V., Chernykh N. I. On the $L_{2}$-approximation of periodic functions by trigonometric polynomials // Approximation and Function Spaces. Proc. Conf. Gdan'sk, 1979. Amsterdam: North-Holland, 1981. P. 25-43.

[6] Бердышев В. И. О теореме Джексона в $L_{p} / /$ Тр. МИАН. 1967. Т. 88. С. 3-16.

[7] Юдин В.А. Одна экстремальная задача для функции распределения // Матем. заметки. 1998. Т. 63. № 2. С. 316-320.

[8] Бабенко А. Г. О точной константе в неравенстве Джексона в $L^{2} / /$ Матем. заметки. 1986. T. 39. № 5. C. $651-664$.

[9] Арестов В. В., Попов В. Ю. Неравенства Джексона на сфере в $L_{2} / /$ Изв. вузов. Матем. 1995. № 8 (399). C. 13-20.

[10] Berdysheva E.E. Several related extremal problems for multivariate entire functions of exponential type // East J. Approx. 2000. V. 6. № 2. P. 241-260.

[11] Arestov V.V., Babenko A. G. Continuity of the best constant in the Jackson inequality in $L^{2}$ with respect to argument of modulus of continuity // Approximation Theory: A volume dedicated to B. Sendov / ed. B. Bojanov. Sofia: DARBA, 2002. P. 13-23.

[12] Dunford N., Schwartz J. T. Linear Operators. Part I: General Theory. Pure and Applied Mathematics. V. 7. New York-London: Interscience Publ., 1958; Reprint. New York: John Wiley, 1988.

[13] Stein E. M., Weiss G. Introduction to Fourier Analysis on Euclidean Spaces. Princeton Math. Series. V. 32. Princeton: Princeton Univ. Press, 1971. 